\title{
Demographic Characteristics and Transmission Risk Factors of Patients with Hepatitis C Virus in Turkey: The EPI-C, A Multicenter and Cross-sectional Trial
}

\author{
Türkiye'de Hepatit C Hastalarının Demografik Karakteristikleri ve Bulașma Risk Faktörleri: \\ Çok Merkezli ve Kesitsel EPI-C Çalıșması
}

\author{
(1) Fehmi Tabak¹, (1) Göktuğ Şirin², (1) Mehmet Demir³, (1) Murat Aladağ4 , (1) Şua Sümer ${ }^{5}$,

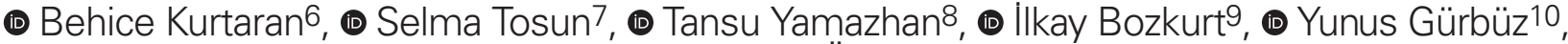 \\ ๑ A Ayşe Batırel11, ๑ Ebubekir Senateș ${ }^{12}$, ๑ Fatma Özlem Kandemir13, ๑ Firdevs Topal14,

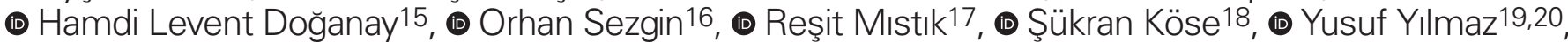 \\ ๑ Dilara Inan21, ๑ Iftihar Köksal22, ๑ Emine Parlak23, ๑ Meral Akdoğan24, ๑ Rahmet Güner25 \\ 1/stanbul University-Cerrahpaşa, Cerrahpaşa Faculty of Medicine, Department of Infectious Diseases and Clinical Microbiology, Istanbul, Turkey \\ 2Kocaeli University Research and Application Hospital, Clinic of Gastroenterology, Kocaeli, Turkey \\ 3 Hatay Mustafa Kemal University Hospital, Clinic of Gastroenterology, Hatay, Turkey \\ 4 Inönü University Turgut Özal Medical Center, Department of Gastroenterology, Malatya, Turkey \\ 5 Selçuk University Faculty of Medicine, Department of Infectious Diseases, Konya, Turkey \\ ${ }^{6}$ Çukurova University Faculty of Medicine, Department of Infectious Diseases and Clinical Microbiology, Adana, Turkey \\ 7 University of Health Sciences Turkey, Izmir Bozyaka Training and Research Hospital, Clinic of Infectious Diseases, Izmir, Turkey \\ ${ }^{8}$ Ege University Faculty of Medicine, Department of Infectious Diseases and Clinical Microbiology, Izmir, Turkey \\ ${ }^{9}$ Ondokuz Mayıs University Faculty of Medicine, Department of Infectious Diseases and Clinical Microbiology, Samsun, Turkey \\ 10 University of Health Sciences Turkey, Dışkapı Yıldırım Beyazıt Training and Research Hospital, Clinic of Infectious Diseases and Microbiology, Ankara, \\ Turkey \\ 11 University of Health Sciences Turkey, Istanbul Kartal Dr. Lütfi Kırdar Training and Research Hospital, Clinic of Infectious Diseases, Istanbul, Turkey \\ 12Istanbul Medeniyet University, Göztepe Training and Research Hospital, Clinic of Infectious Diseases, Istanbul, Turkey \\ 13Mersin University Medical Faculty Hospital, Training and Research Hospital, Clinic of Infectious Diseases, Mersin, Turkey \\ ${ }^{14}$ Izmir Katip Çelebi University, Atatürk Training and Research Hospital, Clinic of Gastroenterology, Izmir, Turkey \\ 15University of Health Sciences Turkey, Ümraniye Training and Research Hospital, Clinic of Gastroenterology, Istanbul, Turkey \\ ${ }^{16}$ Mersin University Health Research and Application Hospital, Clinic of Gastroenterology, Mersin, Turkey

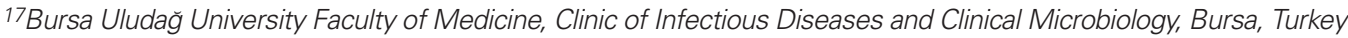 \\ 18University of Health Sciences Turkey, Izmir Tepecik Training and Research Hospital, Clinic of Infectious Diseases and Clinical Microbiology, Izmir, Turkey \\ 19 Marmara University Institute of Gastroenterology, Unit of Liver Research, Istanbul, Turkey \\ 20 Marmara University Faculty of Medicine, Department of Gastroenterology, Istanbul, Turkey \\ ${ }^{21}$ Akdeniz University Hospital, Clinic of Infectious Diseases and Clinical Microbiology, Antalya, Turkey \\ ${ }^{22}$ Karadeniz Technical University, Farabi Hospital, Clinic of Infectious Diseases and Clinical Microbiology, Trabzon, Turkey \\ ${ }^{23}$ Atatürk University Faculty of Medicine, Department of Infectious Diseases and Clinical Microbiology, Erzurum, Turkey \\ 24 Yüksek Ihtisas University Training and Research Hospital, Clinic of Gastroenterology, Ankara, Turkey \\ ${ }^{25}$ Ankara Yıldırım Beyazıt University, Ankara City Hospital, Clinic of Infectious Diseases and Clinical Microbiology, Ankara, Turkey
}

Address for Correspondence: Fehmi Tabak MD, Istanbul University-Cerrahpaşa, Cerrahpaşa Faculty of Medicine, Department of Infectious Diseases and Clinical Microbiology, Istanbul, Turkey Phone: +90 2124143095 E-mail: fehmitabak@hotmail.com ORCID ID: orcid.org/0000-0001-8632-2825 Received: 19.01.2021 Accepted: 21.09.2021

${ }^{\circ}$ Copyright 2021 by Viral Hepatitis Society / Viral Hepatitis Journal published by Galenos Publishing House. 


\begin{abstract}
Objectives: To describe the prevalence of risk factors in patients infected with hepatitis $\mathrm{C}$ virus (HCV).

Materials and Methods: Patients who were aged $>18$ years visiting outpatient clinics and diagnosed as having HCV infection were enrolled in this cross-sectional, multicenter study conducted in 71 cities. Patient data on socio-demographic and clinical characteristics and pre-defined risk factors were collected.

Results: Among 1,018 patients, 53.0\% were women. The mean age was $57.2 \pm 14.3$ years and $34.8 \%$ had been diagnosed as having $\mathrm{HCV}$ infection $>10$ years before enrollment. Almost half of the patients $(45.5 \%)$ were diagnosed during their regular check-up visits, and only $16.8 \%$ were diagnosed because of signs or symptoms of HCV. Genotype 1 and sub-genotype 1 b were detected in $87.9 \%$ and $73.7 \%$ of the patients, respectively. At least one risk factor was present in $94.8 \%$ of the patients. The most frequently reported risk factor was major dental procedures $(79.2 \%)$, followed by major surgical operations (56.9\%) and minor surgical interventions (42.3\%).

Conclusion: Our results revealed that most of the patients with $\mathrm{HCV}$ infection underwent major dental procedures.

Keywords: Hepatitis C, demography, risk factors, Turkey
\end{abstract}

öz

Amaç: $\mathrm{Bu}$ çalışmanın amacı hepatit $\mathrm{C}$ virüsü $(\mathrm{HCV})$ ile enfekte hastalarda risk faktörlerinin prevalansını belirlemektir.

Gereç ve Yöntemler: Poliklinik ziyaretlerinde HCV tanısı almış, 18 yaşından büyük hastalar, 71 farklı ilde yürütülmüş olan bu çok merkezli, kesitsel çalışmaya dahil edilmiştir. Hastaların sosyodemografik ve klinik karakteristikleri ile önceden belirlenmiş olan risk faktörleri ile ilgili veriler toplanmıştır.

Bulgular: Bu çalışmaya dahil edilen toplam 1.018 hastanın \%53,0'ı kadındı. Ortalama yaş 57,2 $\pm 14,3$ yıl idi ve hastaların \%34,8'i için HCV enfeksiyonu tanı süresi $>10$ yıl idi. Hastaların neredeyse yarısı $(\% 45,5)$ rutin check-up sırasında teşhis edildi ve sadece $\% 16,8$ 'i HCV ile ilişkili bir belirti veya semptom nedeniyle teşhis edildi. Olguların \%87,9 ve \%73,7'sinde sırasıyla genotip 1 ve alt genotip 1-b tespit edildi. Hastaların \%94,8'inde en az bir risk faktörü mevcuttu. En sık bildirilen risk faktörü majör dental işlemler $(\% 79,2)$ idi, bunu \%56,9'luk oranla majör cerrahi operasyonlar ve \%42,3'lük oranla minör cerrahi müdahaleler izledi.

Sonuç: Sonuçlarımı, HCV enfeksiyonu olan hastaların çoğunun majör dental işlemler gördüğünü göstermektedir.

Anahtar Kelimeler: Hepatit C, demografi, risk faktörleri, Türkiye

Tabak F, Şirin G, Demir M, Aladağ M, Sümer Ş, Kurtaran B, Tosun S, Yamazhan T, Bozkurt İ, Gürbüz Y, Batırel A, Şenateş E, Kandemir FÖ, Topal F, Doğanay HL, Sezgin O, Mıstık R, Köse Ş, Yılmaz Y, İnan D, Köksal İ, Parlak E, Akdoğan M, Güner R. Demographic Characteristics and Transmission Risk Factors of Patients with Hepatitis C Virus in Turkey: The EPI-C, A Multicenter and Cross-sectional Trial. Viral Hepat J. 2021;27:109-117.

\section{Introduction}

Hepatitis C virus (HCV) can cause both acute and chronic hepatitis, ranging in severity from a mild to a serious, lifelong illness (1). According to recent estimates, more than 71 million people around the world have been infected with $\mathrm{HCV}$, and the mortality rate is 350,000 deaths/year. Despite the high prevalence of the disease, most people infected with this virus are unaware of their infection (2).

Risk factors may contribute to the prevalence of $\mathrm{HCV}$ in different proportions within countries because they vary according to each society's cultural values and lifestyles, as well as geographic and demographic differences (3). Thus, risk factors for $\mathrm{HCV}$ infection should be defined according to the current demographic characteristics and prioritized according to national characteristics. Even though $\mathrm{HCV}$-associated risk factors were previously investigated in Turkey, most of these studies were single-center studies and might not reflect national data $(4,5)$. A multicenter study was conducted in Turkey; however, this retrospective study evaluated sustained virologic response rates achieved by dual therapy in treatment-naïve patients with $\mathrm{HCV}$ and did not evaluate HCV-related risk factors (6). Here, we describe the prevalence of risk factors in patients infected with HCV across Turkey.

Challenges arose stemming from the absence of national epidemiologic studies on HCV in Turkey and the lack of robust data on the regional distribution of the disease based on the geographic definitions within the country. This study was planned to contribute data regarding the regional distribution of HCV infection and provide additional data to the current national database for patients infected with $\mathrm{HCV}$ and the joint activities of societies and associations linked to liver diseases in Turkey because there has been no similar study performed in Turkey.

\section{Materials and Methods}

\section{Study Subjects and Clinical Protocol}

The study was initiated on May $2^{\text {nd }}, 2017$, after obtaining Ethics Committee Istanbul University-Cerrahpaşa, Cerrahpaşa Faculty of Medicine approval in conformation with the Declaration of Helsinki and conducted between June $5^{\text {th }}$ and December $28^{\text {th }}$, 2017 (approval number: D-02, date: 12.07.2016). Infectious disease speciality and gastroenterology sites were selected based on their patient population and their ability to appropriately conduct the study. Patients who were aged 18 years or older at the time of enrollment, diagnosed as having HCV through anti-HCV and HCV-RNA-positive test results, and attending routine visits to their healthcare providers were enrolled. All patients signed an informed consent form before their inclusion in the study. The patients' socio-demographic status, HCV signs and symptoms, laboratory findings (anti-HCV antibody positivity, HCV-RNA level, and HCV genotype), duration of HCV infection, HCV risk factors (e.g., history of surgical operations, organ transplantation, blood transfusion) and comorbidities [e.g., human immunodeficiency virus $(\mathrm{HIV})$, hepatitis $\mathrm{B}$ virus $(\mathrm{HBV})$, diabetes] were recorded. The investigators chose $\mathrm{HCV}$ risk factors from the definition of populations with high HCV prevalence, which was provided by the World Health Organization (WHO) [April 2014 version of the current guideline (1)], and classified these factors according to the local population characteristics. Interventions such as angiography, piercing, tattooing, and circumcision were defined as minor surgical operations (4), and other surgical interventions such as intraabdominal and intrathoracic surgery were considered as major surgical operations (7). Tooth extractions, implants, endodontic 
surgery (e.g. root canal surgery) and periodontal therapy were considered as major dental procedures (8). The primary endpoint of the study was the prevalence of specified risk factors present in patients with $\mathrm{HCV}$.

\section{Study Design}

This cross-sectional, multicenter study was performed in a single visit in different geographic regions of Turkey to describe characteristics of an HCV-infected population and to evaluate the prevalence of risk factors. Each site enrolled patients consecutively until the number of patients allocated to that site was reached. Among the 81 cities located in Turkey, at least one patient was enrolled from 71 cities. Patients who attended routine clinic visits were consecutively included in the study.

\section{Statistical Analysis}

The sample size calculation was based on the frequency of patient-reported risk factors in patients with $\mathrm{HCV}$. Considering the frequency of dental interventions at $68 \%$ (5), with a precision of $3 \%$ and $95 \%$ confidence interval, at least 929 patients needed to be included in the study, thus the inclusion of 1,000 patients was deemed appropriate.

Data from all clinical assessments were summarized using mean, standard deviation (SD), and percentages. For comparison of categorical data for two or more groups, a chi-square test was used. The normality assumption was tested for all continuous variables using the Kolmogorov-Smirnov test. Continuous variables were analyzed using Student's t-test or the Mann-Whitney U test in condition normal/abnormal distribution. Missing values were not interpolated, and no sensitivity analysis was planned. All analyses were performed using the SPSS for Windows, Version 16.0. Chicago, SPSS Inc.

\section{Results}

A total of 1018 patients were enrolled in the study. Female patients constituted $53.0 \%$ of the study population $(n=540)$. The patients' age ranged between 18 to 91 years, and the mean age ( \pm SD) at inclusion was $57.2 \pm 14.3$ years. Most of the patients were living with their parents or family members (58.3\%); $10.2 \%$ were living alone. Almost half of the study population were primary school graduates (Table 1).

Almost one-third of the patients [ $n=354(34.8 \%)]$ had received an $\mathrm{HCV}$ diagnosis $\geq 10$ years before the date of data collection, and $20.7 \%(n=211)$ were diagnosed from 5 to 10 years before that date. Almost half of the patients $[n=474(45.5 \%)]$ were diagnosed during a regular check-up, and only $16.8 \%(n=175)$ were diagnosed during a visit due to signs and/or symptoms of HCV. Among those, the most frequently observed signs and symptoms were clinical symptoms, such as fever, fatigue, and decreased appetite [ $n=104$ $(59.4 \%)]$, and extra-hepatic findings [ $n=53(30.3 \%)]$.

All patients enrolled in the study were $\mathrm{HCV}$ antibody-positive and HCV-RNA-positive. Genotype 1 had a high distribution ratio [n=895 (87.9\%)], and HCV subtype 1-b was the most frequent subtype in the study population $[n=750$ (73.7\%); Table 2].

The most frequently reported comorbidities were diabetes mellitus [ $\mathrm{n}=195$ (19.2\%)] and chronic renal failure requiring dialysis [n=95 (9.3\%)]. Other concomitant diseases included chronic obstructive pulmonary disease and HBV (Table 1).

\begin{tabular}{|c|c|}
\hline Patient demographics & $n=1018$ \\
\hline \multicolumn{2}{|l|}{ Sex } \\
\hline Female & $540(53.0)$ \\
\hline Male & $478(47.0)$ \\
\hline \multicolumn{2}{|l|}{ Aget, years } \\
\hline Mean \pm SD & $57.2 \pm 14.3$ \\
\hline Min.-max. & $18-91$ \\
\hline \multicolumn{2}{|l|}{ Patient residence } \\
\hline City/town & $829(81.4)$ \\
\hline Village/rural area & $186(18.3)$ \\
\hline $\begin{array}{l}\text { Closed community areas (incarcerated, hospices, } \\
\text { military units) }\end{array}$ & $2(0.2)$ \\
\hline Other & $1(0.1)$ \\
\hline \multicolumn{2}{|l|}{ Living situation } \\
\hline With parents/family members & $593(58.3)$ \\
\hline With spouse/partner (with or without children) & $315(30.9)$ \\
\hline Alone & $104(10.2)$ \\
\hline $\begin{array}{l}\text { With others (e.g. dormitory, retirement home, } \\
\text { jails) }\end{array}$ & $6(0.6)$ \\
\hline \multicolumn{2}{|l|}{ Education levels } \\
\hline Higher education, $>18$ years education & $23(2.3)$ \\
\hline University, $14-18$ years education & $94(9.2)$ \\
\hline Secondary school, 6-13 years education & $298(29.3)$ \\
\hline Primary school, 1-5 years education & $448(44.0)$ \\
\hline No formal education & $151(14.8)$ \\
\hline Unknown & $4(0.4)$ \\
\hline \multicolumn{2}{|l|}{ Primary occupation } \\
\hline Student & $13(1.3)$ \\
\hline Employed/self-employed & $212(20.8)$ \\
\hline Retired & $337(33.1)$ \\
\hline Unemployed; not working for pay & $456(44.8)$ \\
\hline \multicolumn{2}{|l|}{ Family income, monthly } \\
\hline No income & $111(10.9)$ \\
\hline$<1500 \mathrm{TL}$ & $327(32.1)$ \\
\hline $1500-3000 \mathrm{TL}$ & $387(38.0)$ \\
\hline$>3000 \mathrm{TL}$ & $142(13.9)$ \\
\hline No answer & $51(5.0)$ \\
\hline \multicolumn{2}{|l|}{ Comorbidity } \\
\hline HIV & $2(0.2)$ \\
\hline Hepatitis B infection & $47(4.6)$ \\
\hline Chronic renal failure requiring dialysis & $95(9.3)$ \\
\hline Diabetes & $195(19.2)$ \\
\hline Disorder requiring immunomodulatory treatment & $32(3.1)$ \\
\hline $\begin{array}{l}\text { Blood disorder requiring regular blood } \\
\text { transfusion }\end{array}$ & $27(2.7)$ \\
\hline Chronic obstructive pulmonary disease & $72(7.1)$ \\
\hline \multicolumn{2}{|c|}{$\begin{array}{l}\text { Data are } \mathrm{n}(\%) \text { unless otherwise stated. }{ }^{\dagger}: \text { Age at the date of signing informe } \\
\text { consent. SD: Standard deviation, Min.: Minimum, max.: Maximum, HIV: Humar } \\
\text { immunodeficiency virus }\end{array}$} \\
\hline
\end{tabular}


At least one risk factor was present in $94.8 \%$ of the patients. Although more than half of the patients [n=579 (56.9\%)] had a major surgical operation as an $\mathrm{HCV}$ risk factor, only $4.2 \%$ $(\mathrm{n}=43)$ had undergone organ transplantation. Additionally, minor surgical operations were reported in $42.3 \%(n=431)$ of the study population, and $37.2 \%(n=379)$ received a blood transfusion or blood products (Figure 1).

The secondary endpoint of the study was to determine the frequency of pre-specified risk factors according to the age and sex of the patients and duration of infection. The majority of the patients $[n=741(72.8 \%)]$ were aged 35 to 69 years. Within this group, most of the patients underwent a major dental procedure $(81.9 \%)$ or had a major surgical intervention (58.3\%). IV/intranasal illicit drug abuse was detected as a significant risk factor in patients aged 18 to 34 years [ $n=44(45.4 \%)]$, higher than in the other age groups $(p<0.05)$. Risk factors such as organ transplant, receiving a blood transfusion or blood products, having a sexual partner with $\mathrm{HBV}, \mathrm{HCV}$, or HIV were more frequent in the 35 to 69 years' age group ( $\mathrm{p}<0.05)$. For transmission of $\mathrm{HCV}$, major surgical operations and major dental procedures were determined as significant risk factors for patients aged $>70$ years $(p<0.05$; Table 3$)$.

Risk factors such as major surgical/dental procedures and a sexual partner with HBV were more common in female patients, whereas minor surgical interventions, organ transplant, sharing personal hygiene equipment (e.g. toothbrush, razor), intravenous/ intranasal illicit drug use, and having multiple sexual partners were more frequently seen in male patients $(p<0.05)$. No sex difference was detected for risk factors such as receiving a blood transfusion or blood products, encountering any blood or bodily fluids, sexual partner with HCV, and sexual partner with HIV ( $p>0.05$; Table 4).

The duration of infection was $<5$ years for the majority of the patients. Major surgical operations, organ transplant,

\begin{tabular}{|c|c|}
\hline & Patients, n (\%) \\
\hline \multicolumn{2}{|l|}{ Genotype } \\
\hline 1 & $895(87.9)$ \\
\hline 2 & $19(1.9)$ \\
\hline 3 & 36 (3.5) \\
\hline 4 & $13(1.3)$ \\
\hline 5 & $1(0.1)$ \\
\hline Unknown & $54(5.3)$ \\
\hline \multicolumn{2}{|c|}{ Sub-genotype } \\
\hline $1-b$ & $750(73.7)$ \\
\hline $1-a$ & $79(7.8)$ \\
\hline 1-unknown & $64(6.3)$ \\
\hline $1-c$ & $1(0.1)$ \\
\hline 1-d & $1(0.1)$ \\
\hline 3-unknown & $18(1.8)$ \\
\hline 3-a & $17(1.7)$ \\
\hline $3-b$ & $1(0.1)$ \\
\hline 2-unknown & $12(1.2)$ \\
\hline $2-a$ & $5(0.5)$ \\
\hline $2-b$ & $2(0.2)$ \\
\hline 4-unknown & $9(0.9)$ \\
\hline 4-a & $2(0.2)$ \\
\hline 4-c & $1(0.1)$ \\
\hline $4-d$ & $1(0.1)$ \\
\hline $5-x$ & $1(0.1)$ \\
\hline
\end{tabular}

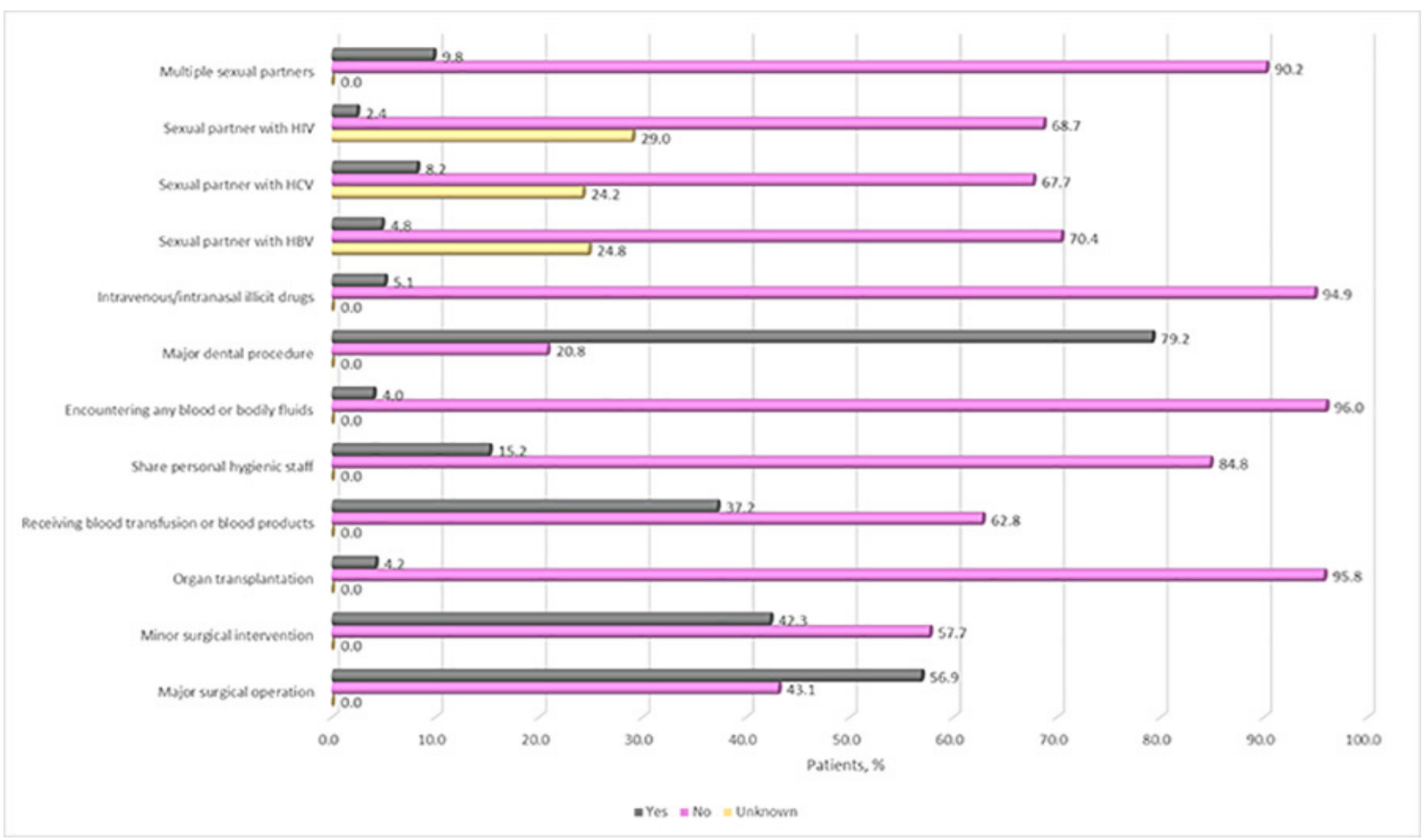

Figure 1. Pre-determined risk factors and distribution of their prevalence HIV: Human immunodeficiency virus, HCV: Hepatitis C virus, HBV: Hepatitis B virus 


\begin{tabular}{|c|c|c|c|c|c|c|c|c|c|c|c|c|c|c|}
\hline & \pm & & ס̊ & స్ి & \%ัٌ & $\begin{array}{l}\bar{o} \\
\dot{i} \\
\dot{v}\end{array}$ & 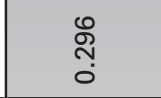 & $\begin{array}{l}9 \\
\stackrel{9}{0} \\
0\end{array}$ & $\begin{array}{l}\bar{o} \\
\dot{v} \\
\dot{v}\end{array}$ & $\begin{array}{l}\bar{o} \\
\dot{i} \\
\dot{v}\end{array}$ & $\begin{array}{l}\bar{o} \\
\dot{0} \\
\dot{v}\end{array}$ & $\begin{array}{l}\bar{o} \\
\dot{v} \\
\dot{v}\end{array}$ & \%̊̊. & \\
\hline & & 旁 & ' & ' & T & ' & ' & ' & ' & 1 & $\begin{array}{l}\widehat{\sigma} \\
\stackrel{\leftrightarrow}{\mathfrak{d}} \\
\stackrel{0}{o}\end{array}$ & 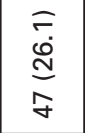 & 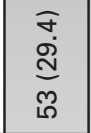 & \\
\hline & & io & 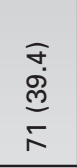 & $\begin{array}{l}\sigma \\
\infty \\
0 \\
0 \\
\varnothing \\
\varnothing \\
\end{array}$ & $\begin{array}{l}\sigma \\
\infty \\
\infty \\
o \\
\infty \\
\stackrel{0}{\sigma} \\
\end{array}$ & $\begin{array}{l}\widehat{o} \\
\stackrel{i}{0} \\
\stackrel{m}{=} \\
=\end{array}$ & 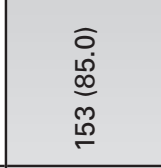 & $\begin{array}{l}\widehat{N} \\
\stackrel{a}{a} \\
\frac{1}{\circ} \\
\therefore\end{array}$ & 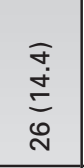 & 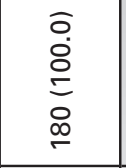 & 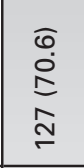 & 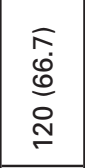 & $\begin{array}{l}\widehat{m} \\
0 \\
\stackrel{0}{0} \\
\stackrel{N}{\sim}\end{array}$ & \\
\hline & & 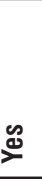 & $\begin{array}{l}\widehat{0} \\
\dot{0} \\
0 \\
\circ \\
\end{array}$ & 亲 & 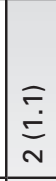 & $\begin{array}{l}\widehat{N} \\
\stackrel{\omega}{m} \\
\widehat{\sigma}\end{array}$ & $\begin{array}{l}\widehat{\widehat{D}} \\
\stackrel{\dot{\rho}}{=} \\
\stackrel{N}{N}\end{array}$ & 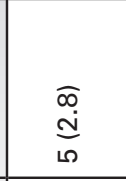 & 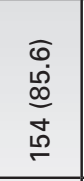 & & $\begin{array}{l}\sigma \\
\stackrel{\sigma}{n} \\
r\end{array}$ & $\begin{array}{l}\widehat{N} \\
\stackrel{v}{*} \\
\underline{m} \\
\end{array}$ & 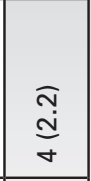 & $\begin{array}{l}\vec{\Phi} \\
\mathbb{\Phi}\end{array}$ \\
\hline \multirow{3}{*}{ 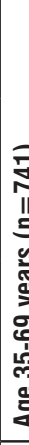 } & & 旁 & . & ' & ' & ' & ' & 1 & 1 & 1 & 옴 & 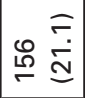 & 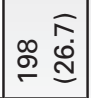 & 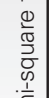 \\
\hline & & $\frac{0}{2}$ & 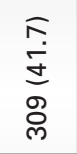 & $\begin{array}{l}\mathcal{J} \\
\infty \\
\stackrel{0}{0} \\
\mathbb{M} \\
\stackrel{y}{y}\end{array}$ & 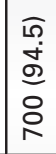 & $\begin{array}{l}\overline{0} \\
0 \\
0 \\
\stackrel{b}{q}\end{array}$ & $\begin{array}{l}\bar{f} \\
\stackrel{\infty}{\infty} \\
\stackrel{\mathscr{m}}{\mathscr{C}}\end{array}$ & 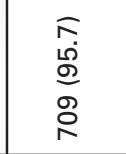 & $\begin{array}{l}\bar{C} \\
\stackrel{0}{\infty} \\
\stackrel{\vec{d}}{c} \\
\stackrel{m}{c}\end{array}$ & $\begin{array}{l}\sigma \\
\infty \\
\stackrel{\infty}{o} \\
\stackrel{m}{\wedge}\end{array}$ & $\begin{array}{l}\frac{1}{10} \\
\stackrel{N}{N} \\
\stackrel{N}{0}\end{array}$ & 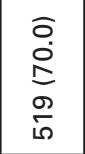 & 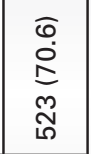 & 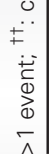 \\
\hline & & 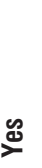 & $\begin{array}{l}\widehat{m} \\
o \\
\infty \\
\stackrel{\rho}{N} \\
\tilde{y}\end{array}$ & 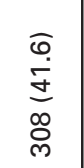 & \begin{tabular}{|l|}
$\underline{10}$ \\
மீ) \\
$\bar{\gamma}$
\end{tabular} & $\begin{array}{l}\bar{D} \\
\stackrel{\rho}{0} \\
\stackrel{0}{0} \\
\stackrel{N}{N}\end{array}$ & 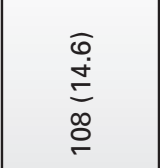 & $\begin{array}{l}\underset{m}{\tilde{j}} \\
\underset{\sim}{\tilde{m}}\end{array}$ & $\begin{array}{l}\frac{\sigma}{\dot{\alpha}} \\
\frac{\sigma}{0} \\
\hat{0}\end{array}$ & $\underset{\infty}{\stackrel{c}{c}}$ & 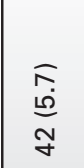 & $\begin{array}{l}\bar{\sigma} \\
\infty \\
\varnothing \\
\varnothing\end{array}$ & $\begin{array}{l}\text { 今 } \\
\text { ํ. } \\
\text { N }\end{array}$ & 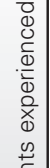 \\
\hline & & $\frac{z}{3}$ & ' & & ' & ' & ' & 1 & & & 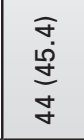 & $\begin{array}{l}\widehat{m} \\
\dot{J} \\
\text { m }\end{array}$ & 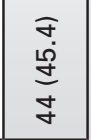 & 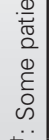 \\
\hline \multirow{2}{*}{ 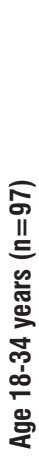 } & & i & $\begin{array}{l}\widehat{0} \\
0 \\
0 \\
8 \\
\circ\end{array}$ & 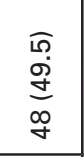 & $\begin{array}{l}0 \\
0 \\
0 \\
0 \\
0 \\
\text { ó }\end{array}$ & 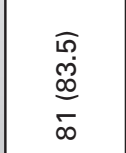 & 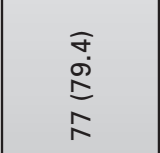 & $\begin{array}{l}\bar{\sigma} \\
\stackrel{\rho}{\circ} \\
\stackrel{\infty}{\infty}\end{array}$ & 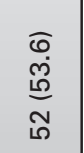 & 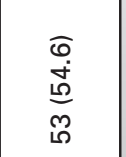 & 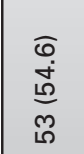 & 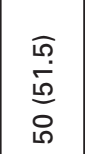 & $\begin{array}{l}\bar{\sigma} \\
\text { D্் } \\
\text { ભ }\end{array}$ & 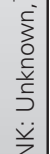 \\
\hline & & 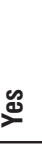 & 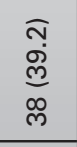 & 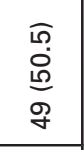 & 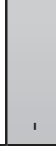 & 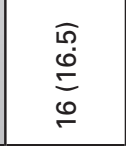 & 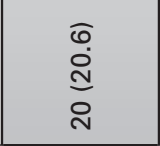 & 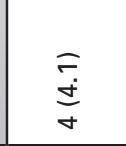 & 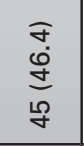 & 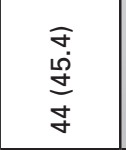 & ' & 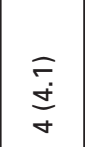 & & 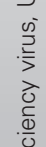 \\
\hline \multirow{4}{*}{ 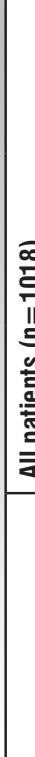 } & & 旁 & ' & & & 1 & ' & ' & & & $\begin{array}{l}\widehat{0} \\
\stackrel{0}{\mathbb{N}} \\
\stackrel{N}{N} \\
\stackrel{N}{N}\end{array}$ & 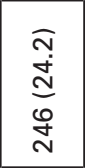 & 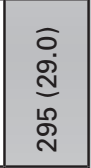 & 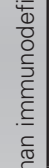 \\
\hline & & $\frac{0}{2}$ & 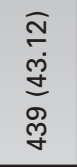 & 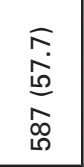 & 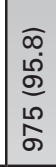 & 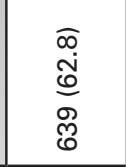 & $\begin{array}{l}\infty \\
\dot{\infty} \\
\infty \\
\dddot{\infty} \\
\infty \\
\infty\end{array}$ & $\begin{array}{l}o \\
\dot{0} \\
\text { o } \\
\text { 合 }\end{array}$ & 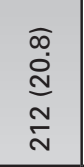 & 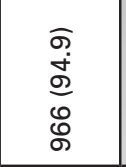 & 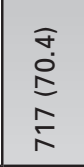 & $\begin{array}{l}\text { i. } \\
\hat{\theta} \\
8 \\
8 \\
0\end{array}$ & $\begin{array}{l}\text { T. } \\
0 \\
0 \\
0 \\
8 \\
0\end{array}$ & 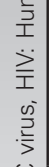 \\
\hline & & ટ્ّ & $\begin{array}{l}\widehat{\sigma} \\
6 \\
0 \\
\sigma \\
\sigma \\
\omega \\
\omega\end{array}$ & 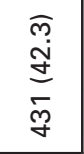 & 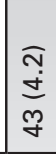 & 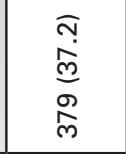 & 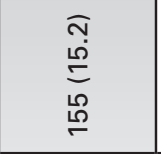 & $\begin{array}{l}\widehat{O} \\
\dot{j} \\
\bar{\gamma}\end{array}$ & $\begin{array}{l}\widehat{N} \\
\stackrel{5}{\Sigma} \\
\stackrel{0}{0} \\
\varnothing \\
\infty\end{array}$ & $\begin{array}{l}\vec{T} \\
\text { ம் } \\
\text { กิ }\end{array}$ & $\begin{array}{l}\widehat{\infty} \\
\dot{d} \\
\stackrel{g}{g}\end{array}$ & $\begin{array}{l}\bar{N} \\
\infty \\
\infty \\
\infty \\
\infty\end{array}$ & 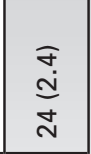 & 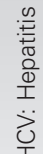 \\
\hline & & 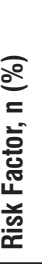 & 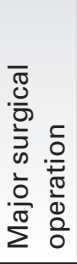 & 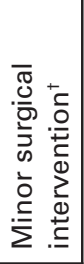 & 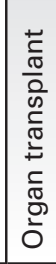 & 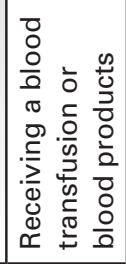 & 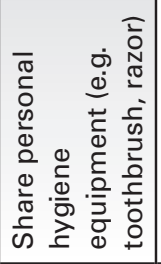 & 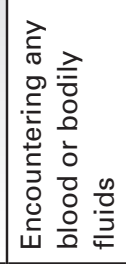 & 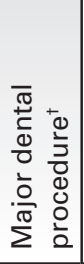 & 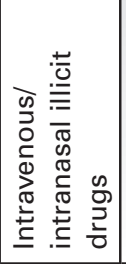 & 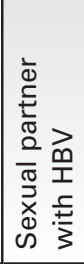 & 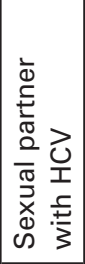 & 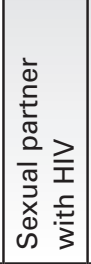 & 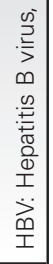 \\
\hline
\end{tabular}




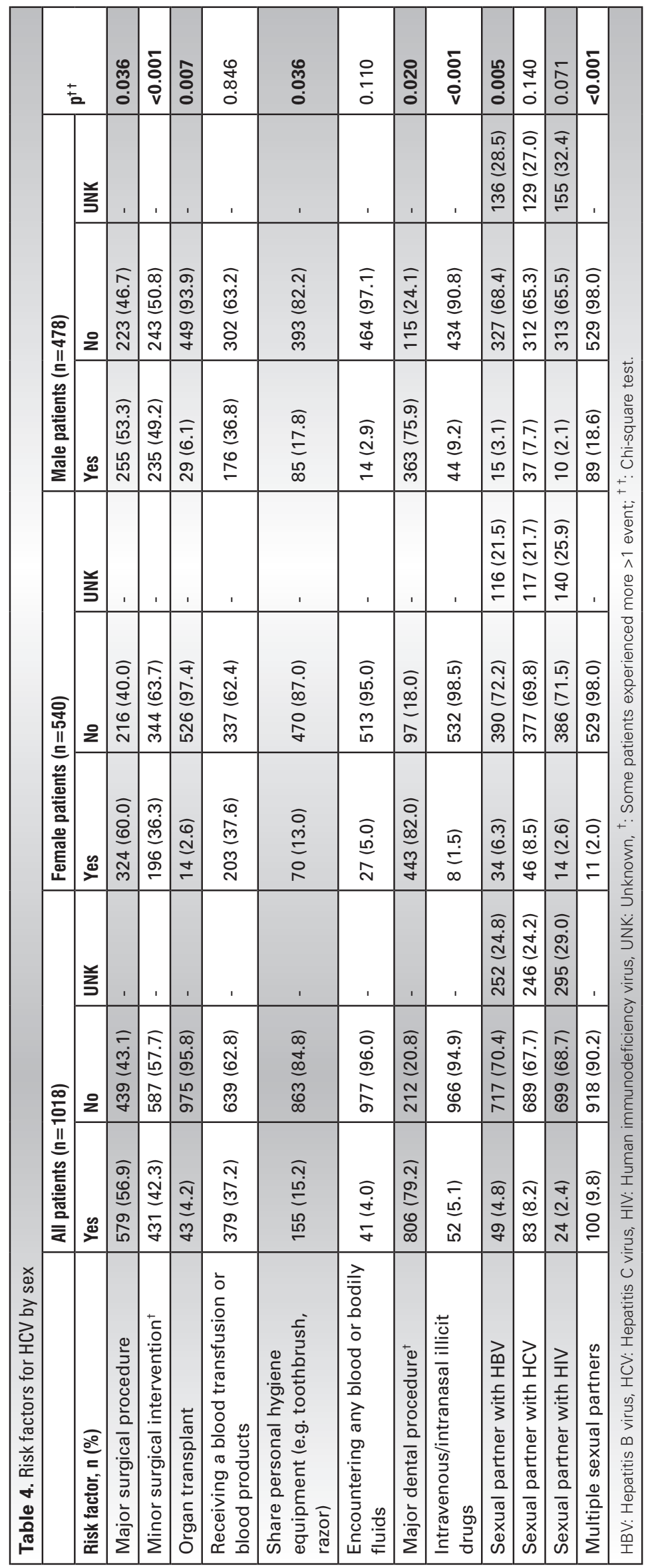

receiving a blood transfusion or blood products, encountering blood or bodily fluids, major dental procedures, and having a sexual partner with $\mathrm{HBV}$, HCV, or HIV were risk factors more frequently detected in the patient group with disease duration of $>10$ years $(p<0.05)$. The use of intravenous/intranasal illicit drugs and having multiple sex partners were more frequent in patients with a shorter duration of disease $(<5$ years; $p<0.05$, Table 5).

\section{Discussion}

This study was designed to explore the prevalence of pre-determined risk factors of $\mathrm{HCV}$ transmission. For the determination of risk factors, the guideline for HCV screening published by the WHO (1) was taken into consideration because population groups with high $\mathrm{HCV}$ prevalence were defined in this guideline. According to the guideline, persons who had undergone interventions such as dental and surgical procedures, blood transfusions, piercing/tattooing or persons who inject drugs were in the high-risk group. Furthermore, the guideline mentioned that these risk factors might vary substantially depending on the geographic region; therefore, recommended risk factors were adopted according to the local setting. As a result, having a surgical procedure (major/ minor), organ transplantation, blood transfusion, major dental procedures, sharing personal hygienic equipment (e.g. toothbrush, razor) at home, encountering blood/body fluids, using IV/intranasal illicit drugs, having a sexual partner with $\mathrm{HCV} / \mathrm{HCV} / \mathrm{HIV}$, and having multiple sexual partners were selected as pre-determined risk factors in this study.

In our study, patients who were HCV-positive had experienced a major surgical operation in 6 of 10 cases, whereas in Poland and Italy, this rate was 38.2\% and 35.6\%, respectively (9). Major dental procedures, which constitute a possible route of $\mathrm{HCV}$ transmission, were experienced by $79.2 \%$ of patients in our study, which was similar to the frequency reported in Poland (79.4\%). A high percentage of patients $(85.7 \%)$ who live in Hatay Province, Turkey, reported major dental procedures as a risk factor, but Italian patients experienced dental procedures less frequently (24.5\%) $(10,11)$. The proportion of patients who experienced a dental and/or surgical procedure was even lower in Greece (10.1\%) (12). One possible reason for the higher proportion of Turkish patients who had surgical and/or dental procedures before receiving an $\mathrm{HCV}$ diagnosis compared with other European countries may be attributed to the differences between health systems and policies, economic situations, and the awareness of infectious diseases. On the other hand, age is a confounding factor for major dental procedures because many oral diseases and conditions are associated with aging. A study conducted in Turkey showed that dental health worsened with increased age (13); therefore, the history of a major dental intervention cannot be considered as a risk factor alone. In Europe, the risk of HCV transmission via medical procedures is lower than the risk of other factors (14), which could be attributed to the greater level of awareness of the medical staff in European countries compared with Turkey. 


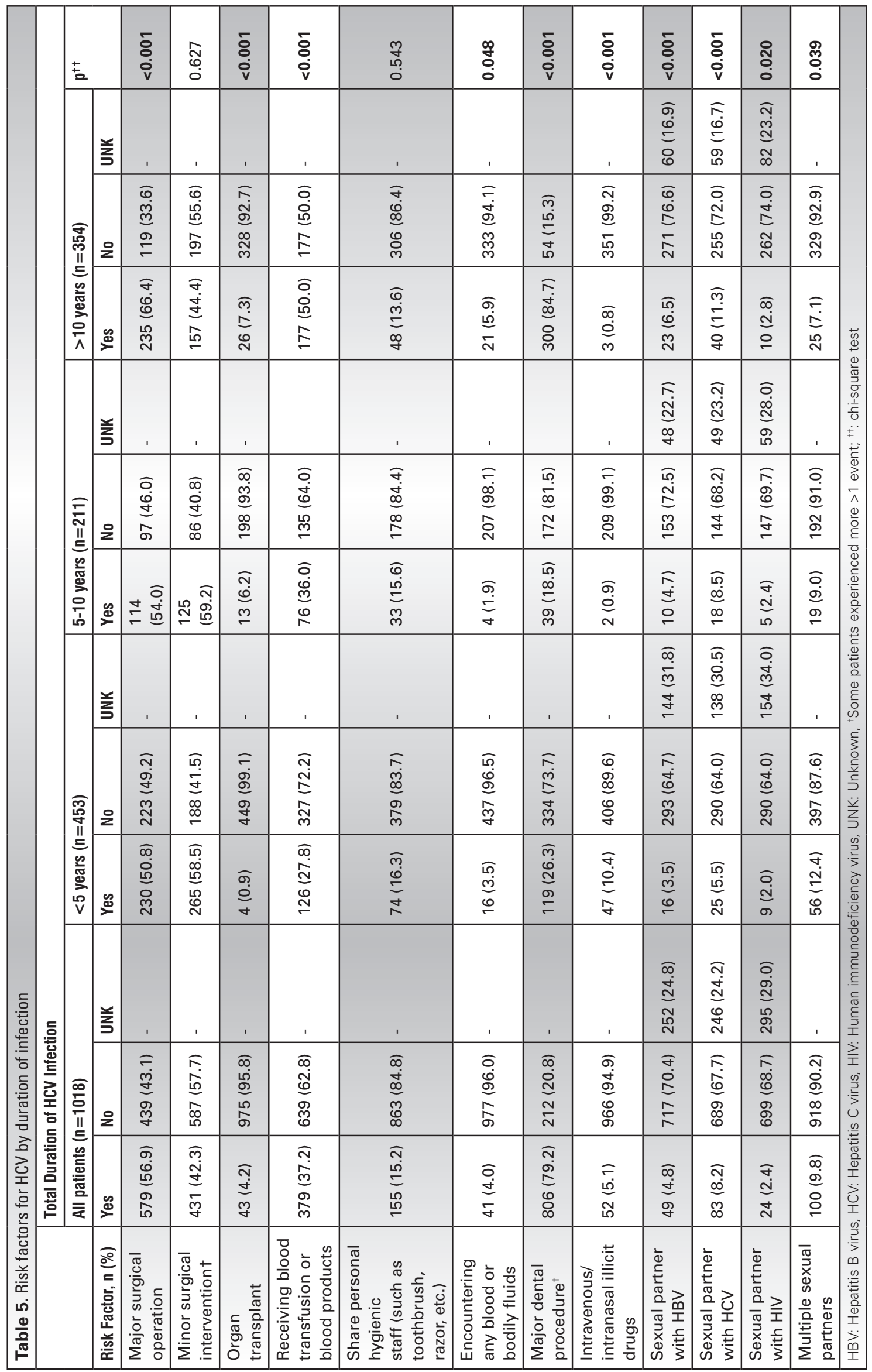


An epidemiologic study conducted in Greece found that blood transfusion was the most common method of HCV transmission (14). In the study by Savvas et al. (15) in 2005, 38.5\% of patients with HCV had a history of blood transfusion; the percentage in our study was similar (37.2\%).

Although HCV can be sexually transmitted, its risk is lower than the risk of other sexually transmitted diseases, such as HBV and HIV. This risk can be decreased from $1 \%$ to $0.6 \%$ or less per year through monogamous relationships. Although the exact prevalence of sexually transmitted HCV between men and women is not known (16), HCV transmission is more frequent in men who have sex with men, especially those who are HIV-positive $(17,18)$. In this study, $9.8 \%$ of patients ( $8.7 \%$ male) had multiple sexual partners, and $8.2 \%$ deliberately ensured that their sexual partner was not $\mathrm{HCV}$ positive. The rate of having HCV-, HBV-, or HIV-positive sexual partners was lower among younger patients than in older patients. Six male patients $(0.6 \%)$ reported having a homosexual relationship.

It has been reported that, worldwide, HCV is transmitted primarily among people who inject drugs $(17,19,20)$, and Gigi et al. (21) reported that $11 \%$ of HCV-positive patients were IV drug users. Additionally, Raptopoulou et al. (12), in their study of patients with HCV IV, reported that drug users were most frequently male. In our national, observational study, IV/intranasal illicit drug abuse was observed in $5.1 \%$ of the study population. Although the prevalence of IV drug abuse in Turkey was lower than in other European countries, it was reported both in the report of European Monitoring Centre for Drugs and Drug Addiction and the Turkish National Drug Report of the Turkish National Police Counter Narcotics Department that IV drug abuse had significantly increased in Turkey $(22,23)$. IV/intranasal illicit drug use was observed mostly among young people compared with other age groups and among male patients compared with female patients in this study. Therefore, to properly define and treat adequately, following-up is especially important in this group of patients.

Patients with chronic HCV infection usually need to change their lifestyle to improve their quality of life. Providing educational programs and counselling services tailored for patients with $\mathrm{HCV}$, especially for those with lower levels of education, may play an important role in the patients changing their lifestyles (24). The impact of HCV on lifestyle was investigated in a study conducted in Italy and the results revealed that $29.5 \%$ of the patients were living alone and the majority of the patients (53.3\%) attended primary school only (24). A similar Korean study in patients with HCV reported that $23.2 \%$ of the patients lived alone and most patients (82.4\%) had not received secondary school education (25). In our study, a lower percentage of patients lived alone when compared with the studies conducted in Italy $(10.2 \%$ vs $29.5 \%)$ and Korea (10.2\% vs $23.2 \%)$. In terms of education levels, we consider our study results to be similar to the results obtained in Italy and Korea.

In a multicenter retrospective study of 1,214 Turkish patients with HCV, 947 were genetically identified, and among those, genotype 1 was the most frequently observed (6). In line with this finding, in our study, the prevalent genotype of the HCV infection was genotype $1-b$, which is similar to global study outcomes published elsewhere (10). Correlatively, genotype 1 was one of the most commonly observed genotypes in European countries (9). According to the results of a study conducted in Italy, the predominant genotype was genotype 1 (63.6\%), followed by genotype 2 (29.4\%). Specifically, genotype 1-b (50.7\%) was the most frequent subtype among those with genotype 1 in the Italian study population (9). Even though the results of the Italian study revealed a sex difference in genotype 1-b (females: 56.4\%, males 44.7\%) and genotype 3 (males: $9.7 \%$, females:2.9\%), we found no sex difference in our study population.

The most frequent comorbidity was diabetes, which is strongly associated with HCV (17). According to the 2016 WHO guidelines, the rates of co-infection of HCV with HIV are high owing to the similarity of their transmission routes, but only two patients were HIV-positive in this study. Additionally, $4.6 \%$ of patients had HBV/ $\mathrm{HCV}$ co-infection, although this co-infection is commonly observed in other regions such as Asia, sub-Saharan Africa, and South Africa, which are HBV-endemic areas (17).

\section{Study Limitations}

One limitation of this study is that, because of the stigmatizing nature of some risk factors, some patients may not have been open to admitting these risk factors. Another limitation may be that, because patients were followed up in special care centers, IV/intranasal illicit drug users were not included in this study. Patient-reported medical history was the only source of information (except HCV-RNA and anti-HCV test results) because this was a non-interventional, exploratory study, The uncertainty of the patients' initial HCV diagnosis dates could be another limitation.

\section{Conclusion}

Even though the most frequently reported risk factor was major dental procedures, it is not possible to consider the history of major dental procedures as a risk factor alone because age is a confounding factor and studies have shown that dental health worsens with increased age. Therefore, additional research is warranted to understand if this is a unique risk factor for $\mathrm{HCV}$ infection. Increasing awareness of viral hepatitis may help to reduce the prevalence of $\mathrm{HCV}$ because transmission of $\mathrm{HCV}$ through all pre-determined risk factors is preventable.

\section{Ethics}

Ethics Committee Approval: The study was initiated on May $2^{\text {nd }}, 2017$, after obtaining Ethics Committee Istanbul University-Cerrahpaşa, Cerrahpaşa Faculty of Medicine approval in conformation with the Declaration of Helsinki and conducted between June $5^{\text {th }}$ and December $28^{\text {th }}, 2017$ (approval number: D-02, date: 12.07.2016).

Informed Consent: All patients signed an informed consent form before their inclusion in the study.

Peer-review: Externally and internally peer-reviewed.

\section{Authorship Contributions}

Concept: Fe.T., G.S.., M.D., Mu.A., S.S., B.K., S.T., T.Y., I.B., Y.G., A.B., E.S.., FÖ.K., Fi.T., H.L.D., O.S., R.M., S..K., Y.Y., D.I., I.K., E.P., Me.A., R.G., Design: Fe.T., G.Ş., M.D., Mu.A., S..S., B.K., S.T., T.Y., I.B., Y.G., A.B., E.Ş., FÖ.K., Fi.T., H.L.D., O.S., R.M., S..K., Y.Y., D.I., I.K., E.P., Me.A., R.G., Data Collection or Processing: Fe.T., G.S., M.D., Mu.A., S.S., B.K., S.T., T.Y., I.B., Y.G., A.B., E.S.., FÖ.K., Fi.T., H.L.D., O.S., R.M., S.K., Y.Y., D.I., I.K., E.P., Me.A., R.G., Analysis or Interpretation: Fe.T., G.Ş., M.D., Mu.A., Ş.S., B.K., S.T., T.Y., I.B., Y.G., A.B., E.Ş., FÖ.K., Fi.T., H.L.D., O.S., R.M., S..K., Y.Y., D.I., I.K., E.P., Me.A., R.G., Literature Search: Fe.T., G.Ş., M.D., Mu.A., Ş.S., B.K., S.T., T.Y., I.B., 
Y.G., A.B., E.Ş., F.̈.K., Fi.T., H.L.D., O.S., R.M., Ş.K., Y.Y., D.I., I.K., E.P., Me.A., R.G., Writing: Fe.T., G.Ş., M.D., Mu.A., S..S., B.K., S.T., T.Y., I.B., Y.G., A.B., E.Ş., FÖ.K., Fi.T., H.L.D., O.S., R.M., S..K., Y.Y., D.I., I.K., E.P., Me.A., R.G.

Conflict of interest: The following authors received research grant and or consulting/speaker fees from the pharmaceutical companies as shown: Fehmi Tabak (Abbvie, Gilead, GSK), Selma Tosun (AbbVie), Tansu Yamazhan (AbbVie, Gilead), Yusuf Yılmaz (AbbVie, Gilead, Abdi Ibrahim, Bilim Ilaç, Nobel), Orhan Sezgin (AbbVie, Gilead, Abdi lbrahim, Bilim, Takeda, Ferring, UCB, Drogsan), Behice Kurtaran (AbbVie, Gilead, Abdi Ibrahim, Pfizer, MSD), Dilara Inan (AbbVie, Gilead, Abdi Ibrahim, Santa Farma), Reşit Mıstık (Gilead), Ebubekir Şenateş (AbbVie, Sanofi, BMS), Fatma Özlem Kandemir (Gilead, Pfizer, MSD), Hamdi Levent Doğanay (BMS, AbbVie, Genfit), Iftihar Köksal (AbbVie, Gilead, Pfizer), Meral Akdoğan (AbbVie, Bayer), Rahmet Güner (Gilead, AbbVie, Pfizer).

The following authors have no conflict of interest to declare: Ayşe Batırel, Emine Parlak, Firdevs Topal, Göktuğ Şirin, Ilkay Bozkurt, Mehmet Demir, Murat Aladağ, Şua Sümer, Şükran Köse, Yunus Gürbüz.

Financial Disclosure: This study was funded in full by AbbVie Inc. (North Chicago, IL, USA). Statistical analysis, writing support, and editing services in the development of this manuscript were provided by MONITOR CRO (Istanbul, Turkey) and funded by AbbVie. The authors also acknowledge the other participants of the EPI-C Study (protocol number 10-989) Team.

The design and study conduct for the EPI-C study were provided by AbbVie. AbbVie participated in the interpretation of data, review, and approval of the publication. All authors received payments from AbbVie to participate in this study.

\section{References}

1. Guidelines for the Screening Care and Treatment of Persons with Chronic Hepatitis C Infection. World Health Organization; 2014

2. Jefferies M, Rauff B, Rashid H, Lam T, Rafiq S. Update on global epidemiology of viral hepatitis and preventive strategies. World J Clin Cases. 2018;6:589-599.

3. European Association for the Study of the Liver. EASL Recommendations on Treatment of Hepatitis C 2018. J Hepatol 2018;69:461-511

4. Yildirim B, Tahan V, Ozaras R, Aytekin H, Mert A, Tabak F, Senturk $\mathrm{H}$. Hepatitis $\mathrm{C}$ virus risk factors in the Turkish community. Dig Dis Sci. 2005;50:2352-2355

5. Barut $S$, Erkorkmaz U, Yüce $S$, Uyetürk U. Analysis of risk factors in anti-HCV positive patients in Gaziosmanpasa University Hospital, Tokat, Turkey. Mikrobiyol Bul. 2008;42:675-680.

6. Gürbüz $Y$, Tülek NE, Tütüncü EE, Koruk ST, Aygen B, Demirtürk N, Kınıkı S, Kaya A, Yıldırmak T, Süer K, Korkmaz F, Ural O, Akhan $S$ Günal Ö, Tuna N, Köse Ş, Gönen I, Örmen B, Türker N, Saltoğlu N, Batırel A, Tuncer G, Bulut C, Sırmatel F, Ulçay A, Karagöz E, Tosun D, Şener A, Aynığlu A, Altunok ES. Evaluation of Dual Therapy in Real Life Setting in Treatment-Naive Turkish Patients with HCV Infection: A Multicenter, Retrospective Study. Balkan Med J. 2016;33:18-26.

7. Yousef MA, Vaida S, Somri M, Mogilner J, Lanir A, Tamir A, Shaou R. Changes in creatine phosphokinase (CK) concentrations after minor and major surgeries in children. Br J Anaesth. 2006;96:786789.

8. Manski RJ, Hyde JS, Chen H, Moeller JF. Differences among older adults in the types of dental services used in the United States. Inquiry. 2016;53:0046958016652523

9. Petruzziello A, Coppola N, Loquercio G, Marigliano S, Giordano M, Azzaro R, Diodato AM, lervolino V, Di Costanzo G, Di Macchia
CA, Di Meo T, Paradiso L, Ferro R, Giuliano P, Russo F, Pasquale G, Cacciapuoti C. Distribution pattern of hepatitis $\mathrm{C}$ virus genotypes and correlation with viral load and risk factors in chronic positive patients. Intervirology. 2014;57:311-318.

10. Messina JP, Humphreys I, Flaxman A, Brown A, Cooke GS, Pybus OG, Barnes E. Global distribution and prevalence of hepatitis $C$ virus genotypes. Hepatology. 2015;61:77-87.

11. Rosińska M, Parda N, Kołakowska A, Godzik P, Zakrzewska K, Madaliński K, Zieliński A, Boguradzka A, Gierczyński R, Stępień M. Factors associated with hepatitis $C$ prevalence differ by the stage of liver fibrosis: A cross-sectional study in the general population in Poland, 2012-2016. PLoS One. 2017;12:e0185055.

12. Raptopoulou M, Touloumi G, Tzourmakliotis D, Nikolopoulou G, Dimopoulou M, Giannoulis G, Vasiliadis T, Skoutelis A, Anagnostou O, Hatzis G, Manolakopoulos S. Significant epidemiological changes in chronic hepatitis $C$ infection: results of the nationwide HEPNETGREECE cohort study. Hippokratia. 2011;15:26-31.

13. Karaaslan F, Dikilitas A, Yiğit U. Oral health status and associated factors in a subpopulation of Turkish patients. Cumhuriyet Dental J. 2019;22:167-175

14. Triantos C, Konstantakis C, Tselekouni P, Kalafateli M, Aggeletopoulou I, Manolakopoulos S. Epidemiology of hepatitis C in Greece. World J Gastroenterol. 2016;22:8094-8102.

15. Savvas SP, Koskinas J, Sinani C, Hadziyannis A, Spanou F, Hadziyannis SJ. Changes in epidemiological patterns of HCV infection and their impact on liver disease over the last 20 years in Greece. J Viral Hepat. 2005;12:551-557.

16. Terrault NA. Sexual activity as a risk factor for hepatitis C Hepatology. 2002;36(Suppl1):S99-105.

17. Guidelines for the Screening Care and Treatment of Persons with Chronic Hepatitis C Infection: Updated Version. Geneva: World Health Organization; 2016.

18. Yaphe S, Bozinoff N, Kyle R, Shivkumar S, Pai NP, Klein M. Incidence of acute hepatitis $C$ virus infection among men who have sex with men with and without HIV infection: a systematic review. Sex Transm Infect. 2012;88:558-564.

19. Grebely J, Robaeys G, Bruggmann P, Aghemo A, Backmund M, Bruneau J, Byrne J, Dalgard O, Feld JJ, Hellard M, Hickman M, Kautz A, Litwin A, Lloyd AR, Mauss S, Prins M, Swan T, Schaefer M, Taylor LE, Dore GJ, International Network for Hepatitis in Substance Users. Recommendations for the management of hepatitis $\mathrm{C}$ virus infection among people who inject drugs. Int J Drug Policy. 2015;26:1028-1038.

20. Papatheodoridis GV, Hatzakis A, Cholongitas E, Baptista-Leite R, Baskozos I, Chhatwal J, Colombo M, Cortez-Pinto H, Craxi A, Goldberg D, Gore C, Kautz A, Lazarus JV, Mendao L, PeckRadosavljevic M, Razavi H, Schatz E, Tozun N, van Damme $\mathrm{P}$ Wedemeyer H, Yazdanpanah Y, Zuure F, Manns MP. Hepatitis C: The beginning of the end-key elements for successful European and national strategies to eliminate HCV in Europe. J Viral Hepat. 2018;25(Suppl1):6-17.

21. Gigi E, Sinakos E, Sykja A, Androulakis G, Tanis C, Stayridou V, Tsirogianni E, Zouridakis K, Bellou AL, Orfanou E, Raptopoulou-Gigi M. Epidemiology, clinical data, and treatment of viral hepatitis in a large cohort of intravenous drug users. J Addict Med. 2013;7:52-57.

22. Sarasa-Renedo A, Barrio G, Montanari L, Guarita B, de la Fuente $\mathrm{L}$, Bravo MJ, Vicente J. Estimating trends in injecting drug use in Europe using national data on drug treatment admissions. Lisbon: European Monitoring Centre for Drugs and Drug Addiction; 2015.

23. Turkey, Country Drug Report 2017. Luxembourg: European Monitoring Centre for Drugs and Drug Addiction; 2017.

24. Scognamiglio P, Galati V, Navarra A, Longo MA, Aloisi MS, Antonini MG, Puoti M, Almasio PL, Ippolito G, Girardi E. Impact of hepatitis C virus infection on lifestyle. World J Gastroenterol. 2007;13:27222726.

25. Cho HJ, Park E. Quality of life of chronic hepatitis c patients and its associated factors. Osong Public Health Res Perspect. 2017;8:124129. 